\title{
Binary fluids under steady shear in three dimensions
}

\author{
K. Stratford ${ }^{1,2}$, J.-C. Desplat ${ }^{3}$, P. Stansell ${ }^{1}$, and M. E. Cates ${ }^{1}$ \\ ${ }^{1}$ SUPA, School of Physics and ${ }^{2}$ EPCC, The University of Edinburgh, \\ JCMB The King's Buildings, Mayfield Road, Edinburgh, EH9 3JZ, \\ United Kingdom; ${ }^{3}$ Irish Centre for High-End Computing, \\ Dublin Institute for Advanced Studies, 5 Merrion Square, Dublin 2, Ireland
}

\begin{abstract}
We simulate by lattice Boltzmann the steady shearing of a binary fluid mixture with full hydrodynamics in three dimensions. Contrary to some theoretical scenarios, a dynamical steady state is attained with finite correlation lengths in all three spatial directions. Using large simulations we obtain at moderately high Reynolds numbers apparent scaling exponents comparable to those found by us previously in 2D. However, in 3D there may be a crossover to different behavior at low Reynolds number: accessing this regime requires even larger computational resource than used here.
\end{abstract}

PACS numbers: $64.75 .+\mathrm{g}, 47.11 . \mathrm{Qr}$

Systems that are not in thermal equilibrium play a central role in modern statistical physics [1]. They include two important classes: those evolving towards Boltzmann equilibrium (e.g., by phase separation following a temperature quench), and those maintained in nonequilibrium by continuous driving (such as a shear flow). Of fundamental interest, and surprising physical subtlety, are systems combining both features — such as a binary fluid undergoing phase separation in the presence of shear. Here a central issue [2, 3] is whether coarsening continues indefinitely, as it does without shear, or whether a nonequilibrium steady state (NESS) is reached, in which the characteristic length scales $L_{x, y, z}$ of the fluid domain structure attain finite $\dot{\gamma}$-dependent values at late times. (We define the mean velocity as $u_{x}=\dot{\gamma} y$ so that $x, y, z$ are velocity, velocity gradient and vorticity directions respectively; $\dot{\gamma}$ is the shear rate.)

Our recent simulations, building on earlier work of oth-

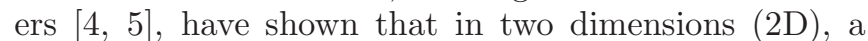
NESS is indeed achieved [6]. In 3D, the situation is more subtle. Fourier components of the composition field whose wavevectors lie along the vorticity direction feel no direct effect of the mean advective velocity [2, 7. Therefore it might be possible for coarsening to proceed indefinitely by pumping through tubes of fluid oriented along $z$ [3]. Another crucial difference is that in 2D fluid bicontinuity is possible only by fine tuning to a percolation threshold at 50:50 composition (assuming fluids of equal viscosity) so that the generic situation is one of droplets. (Indeed, for topological reasons, droplets are implicated even at threshold 伯.) In contrast, in 3D both fluids remain continuously connected across the sample throughout a broad composition window either side of 50:50.

In 3D experiments, saturating length scales are reportedly reached after a period of anisotropic domain growth [2. 8]. However, the extreme elongation of domains along the flow direction means that, even in experiments, finite size effects could play a role in such saturation [9]. Theories in which the velocity does not fluctuate, but does advect the diffusive fluctuations of the concentration field, predict instead indefinite coarsening, with length scales $L_{y, z}$ scaling as $\dot{\gamma}$-independent powers of the time $t$ since quench, and (typically) $L_{x} \sim \dot{\gamma} t L_{y}$ [9]. As emphasized in [6], in real fluids, however, the velocity fluctuates nonlinearly in response to the advected concentration field, and hydrodynamic scaling arguments, balancing interfacial and either viscous or inertial effects, predict saturation instead e.g., $L / L_{0} \sim\left(\dot{\gamma} T_{0}\right)^{-1}$ or $L / L_{0} \sim\left(\dot{\gamma} T_{0}\right)^{-2 / 3}$ [3, 10, 11]. Here, $L_{0}=\nu^{2} /(\rho \sigma)$ and $T_{0}=\nu^{3} /\left(\rho \sigma^{2}\right)$, with $\rho$ density, $\nu=\eta / \rho$ kinematic viscosity and $\sigma$ interfacial tension, are the characteristic length and time at which inertial effects start to influence coarsening [12]. Given these uncertainties as to the fate of sheared binary fluids in $3 \mathrm{D}$, computer simulations of such systems, with full hydrodynamic velocity fluctuations, are of great interest.

Such simulations also offer demanding challenges to the state of the art in computational physics. The 2D lattice Boltzmann (LB) results of [6] were obtained from 16 production runs involving lattices ranging from $512 \times 256$ to $2048 \times 1024$ (all systems having aspect ratio $2: 1$ ). Many pre-production runs were required to steer simulation parameters so as to avoid finite-size effects and other artefacts. This effort was rewarded, however: the unique parametric flexibility of LB allowed us to probe over six decades of reduced shear rate $\dot{\gamma} T_{0}$ [6]. Below, we extend that work to three dimensions with 9 production runs on $512 \times 256 \times 256$ lattices, and 3 larger runs of $1024 \times 512 \times 512$ (i.e., all with aspect ratio 2:1:1). Even given the excellent parallel scaling of LB on multiprocessor machines, each one of these 12 datasets required more computational resource than the entirety of Ref.[6]. The production runs reported here were performed using 1024 processors of the IBM Blue Gene/L machine at the University of Edinburgh.

Although our simulations are not the first to address sheared binary fluids in 3D (see e.g. [3, 13), earlier studies have offered only inconclusive evidence of NESS formation in systems free of finite size effects. Such effects can cause fully lamellar or hexagonal cylindrical domains, which wrap the periodic boundary conditions with simple topologies that prevent further hydrodynamic coarsening 35, 14; but this "trivial" route to NESS relies directly on the periodic boundary conditions and is thus not available in the bulk-system limit. Below we present evi- 


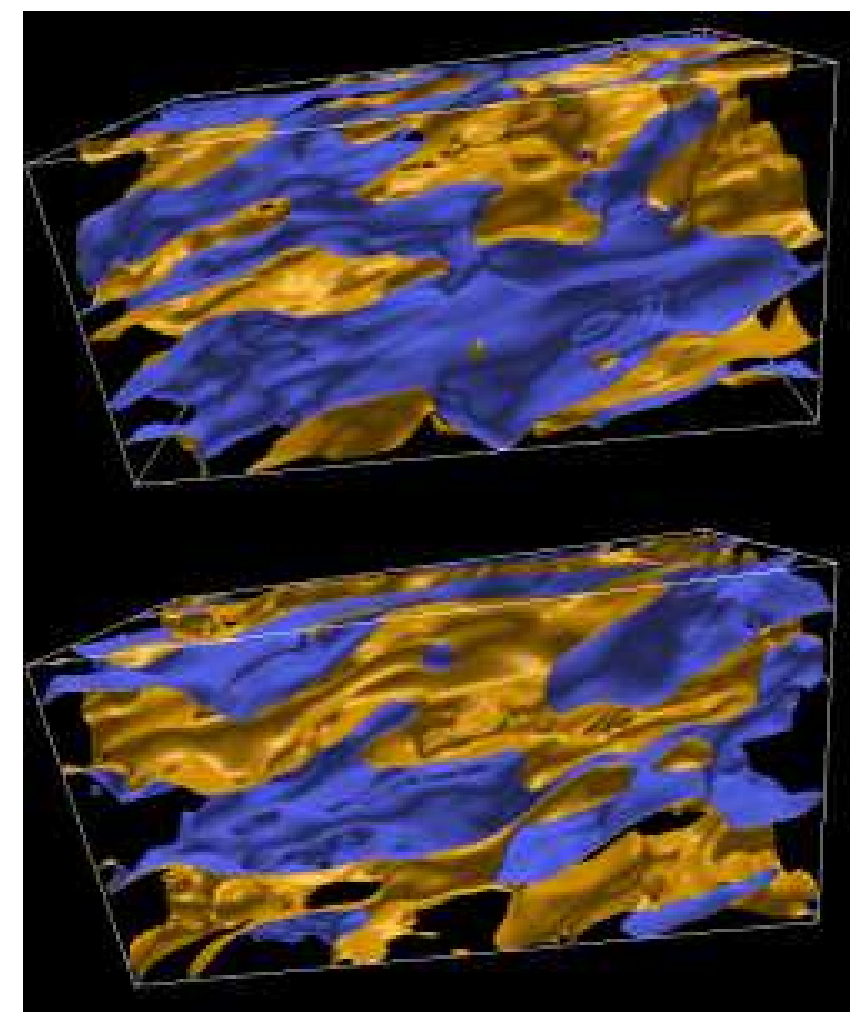

Figure 1: Snapshots of the interface position at $\dot{\gamma} T_{0}=22.47$ (top) and 47.45 (bottom) with parameter set R019 (Table 1). These are representative of the observed NESS. The mean flow is rightward along the upper face of the simulation box and leftward at the lower face; the line of sight lies close to the vorticity (neutral) direction, $z$. Yellow and blue isosurfaces are constructed at $\phi= \pm 0.2$ to create a dividing surface colorcoded by the adjacent fluids (both shown transparent).

dence of NESS formation in systems retaining the complex topology expected in bulk samples, where a steady state dynamical balance can arise between the coarsening of bicontinuous domains under the action of interfacial tension, and their stretching by the flow (Fig.11).

The required parameter steering would not have proven possible without having the $2 \mathrm{D}$ runs to initially guide our selection - a methodology that can only succeed if the physics in $2 \mathrm{D}$ and $3 \mathrm{D}$ is not radically different. Below we find that to be true for the upper few decades of the range of $\left(\dot{\gamma} T_{0}\right)^{-1}$ addressed in [6]; within this range, evidence is given below for saturation of correlation lengths with finite values in all three directions. We then combine datasets using a quantitative scaling methodology developed for the unsheared problem in 12 and for shear in [6]; this allows scaling exponents to be estimated using combined multi-decade fits. Caution is required here due to residual finite size effects; these are unavoidable, particularly at high shear rates where we find NESS hardest to achieve numerically. Note that high shear rates correspond to low Reynolds numbers Re $\simeq L_{y}^{2} \dot{\gamma} / \nu$ (due to the decrease of domain size with shear rate); these results could therefore signify new physics at low Re 3]. However, much larger systems sizes might be needed to gain full access to this regime.

The governing equations for our binary fluid system are the Cahn-Hilliard equation for the composition $\phi$, and the incompressible Navier-Stokes equation for the velocity $u_{\alpha}$ in an isothermal fluid of unit density $\rho$ :

$$
\begin{aligned}
\left(\partial_{t} u_{\alpha}+u_{\beta} \nabla_{\beta} u_{\alpha}\right)+\nabla_{\alpha} p-\nu \nabla^{2} u_{\alpha}-\phi \nabla_{\alpha} \mu & =0(1) \\
\partial_{t} \phi+\nabla_{\alpha}\left(\phi u_{\alpha}-M \nabla_{\alpha} \mu\right) & =0(2)
\end{aligned}
$$

Here, $p$ is pressure (related in LB to density fluctuations, which are small [12]); $\nu$ is the kinematic viscosity; $M$ is the ( $\phi$-independent) mobility and $\mu=B \phi\left(\phi^{2}-1\right)-$ $\kappa \nabla^{2} \phi$ is the chemical potential. $B$ and $\kappa$ are positive constants; the interfacial tension is $\sigma=(8 \kappa B / 9)^{1 / 2}$ and the interfacial width is $\xi_{0}=(2 \kappa / B)^{1 / 2}[12$.

We solve these equations with an LB algorithm similar to that reported in [12, 15. To achieve the necessary shear rates, the domain is decomposed blockwise using multiple Lees-Edwards sliding periodic boundary conditions 6. 16], chosen so that $\int_{0}^{\Lambda_{y}} \nabla_{y} u_{x} d y=\Lambda_{y} \dot{\gamma}$. Although we neglect thermal fluctuations in our fluid, as appropriate for dynamics near a zero-temperature fixed point 17, a fluctuating local velocity field still arises via nonlinear interaction between the order parameter field and flow field. To help control errors, we adhered as far as possible to previously used parameter values and protocols [6, 12]. However, the sheared 3D case showed significant stability problems compared with either the 3D unsheared case or 2D sheared case. To alleviate these, we replaced the D3Q15 lattice of 12 with a D3Q19 model; this removes a "computational mode" responsible for some of the instabilities of D3Q15 [18]. We also use a multiple relaxation time approach 19 in place of a single relaxation time [6, 12, further improving stability.

Most of our 3D production runs were made using system size $512 \times 256 \times 256$, run for $t \simeq 4 \times 10^{5}$ time steps. Holding other parameters fixed, one finds that if $\dot{\gamma}$ is too small, the domain size is large and finite size effects dominate, whereas if $\dot{\gamma}$ is too large then the domains become small on the lattice scale and tend to form a partially (or even fully) remixed state with strongly blurred interfaces. Such remixing could be a real physical effect at shear rates so high that the local interfacial structure departs strongly from equilibrium, but this happens at much lower shear rates in an LB fluid than in a real one (where $\xi_{0}$ is much smaller). We therefore reject as artefacts all such partially remixed states, as identified by a significant reduction in order parameter variance $\left\langle\phi^{2}\right\rangle$. Worst affected were the runs at higher Reynolds number (low viscosity) where an adjustment of the interfacial width from $\xi_{0}=1.13$ to $\xi_{0}=1.35$ helped to maintain acceptable behavior. All simulations reported here were done for fully symmetric quenches with parameters summarized in Table 1. As in the unsheared case 12], judicious combinations of $\xi_{0}, \sigma, M$ and $\nu$ allow systems spanning several decades in $L / L_{0}$ and $\dot{\gamma} T_{0}$ to be accurately studied by varying $L_{0}$ and $T_{0}$ alongside $\dot{\gamma}$.

Fig. 11 shows snapshots of the interfacial structure based on the order parameter field for R019 with $\dot{\gamma}=$ 


\begin{tabular}{ccccccccccccc}
\hline Name & $\nu$ & $M$ & $\sigma_{\text {theory }}$ & $\sigma_{\text {meas }}$ & $L_{0}$ & $T_{0}$ & $\xi_{0}$ & $\dot{\gamma}$ & $\Lambda_{x}$ & $L_{x}$ & $L_{y}$ & $L_{z}$ \\
\hline R028 & 1.41 & 0.05 & 0.063 & 0.055 & 36.1 & 927 & 1.13 & $5.0 \times 10^{-4}$ & 1024 & - & - & - \\
R029 & 0.2 & 0.15 & 0.047 & 0.042 & 0.952 & 4.54 & 1.13 & $5.0 \times 10^{-4}$ & 1024 & - & - & - \\
R020 & 0.025 & 2 & 0.0047 & 0.0042 & 0.149 & 0.886 & 1.13 & $5.0 \times 10^{-4}$ & 1024 & - & - & - \\
R003 & 0.015 & 2.0 & 0.0047 & 0.0042 & 0.054 & 0.19 & 1.13 & $7.5 \times 10^{-4}$ & 512 & 511 & 72.2 & 172 \\
& & & & & & & & $5.0 \times 10^{-4}$ & 512 & 828 & 116 & 352 \\
R004 & 0.01 & 2.0 & 0.0047 & 0.0042 & 0.024 & 0.0567 & 1.13 & $7.5 \times 10^{-4}$ & 512 & 356 & 68.1 & 131 \\
& & & & & & & & $5.0 \times 10^{-4}$ & 512 & 491 & 106 & 192 \\
R030 & 0.00625 & 1.25 & 0.0047 & 0.0042 & 0.00930 & 0.0138 & 1.13 & $5.0 \times 10^{-4}$ & 512 & 375 & 91.6 & 160 \\
R007 & 0.005 & 2.0 & 0.0047 & 0.0042 & 0.0059 & 0.00709 & 1.13 & $5.0 \times 10^{-4}$ & 512 & 382 & 97.4 & 174 \\
R008 & 0.0035 & 2.0 & 0.0047 & 0.0042 & 0.0029 & 0.00243 & 1.35 & $5.0 \times 10^{-4}$ & 512 & 370 & 101 & 177 \\
R019 & 0.0014 & 4 & 0.0024 & 0.0021 & 0.000933 & 0.000622 & 1.35 & $5.0 \times 10^{-4}$ & 512 & 234 & 71.3 & 118 \\
R032 & 0.0005 & 5 & 0.00094 & 0.00083 & 0.000301 & 0.000181 & 1.35 & $5.0 \times 10^{-4}$ & 512 & 135 & 48.0 & 71.2 \\
\hline
\end{tabular}

Table I: Parameter sets used in 3D simulations and observed NESS length scales. Where a trivial NESS could be identified by inspection, no length is recorded. The results of R020 were ambiguous: periods of apparent NESS were contaminated by intervals of partial remixing (low $\left\langle\phi^{2}\right\rangle$ ).

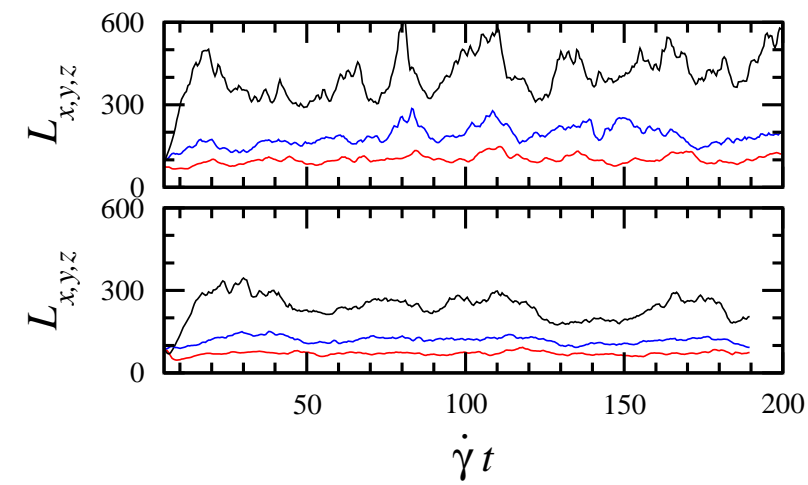

Figure 2: Two examples of $L_{x, y, z}$ in lattice units as a function of time in strain units $\dot{\gamma} t$ for R030 parameters (upper panel) and R019 parameters (lower panel). For all parameters where a steady state is observed, the length scale as measured by the gradient statistic of [4] is largest in the velocity direction $L_{x}$, followed by the vorticity direction $L_{z}$, with that in the velocity gradient direction $L_{y}$ the smallest.

$5 \times 10^{-4}$ after a steady state had been reached [20]. Fig. 目 shows time series for $L_{x, y, z}$ from runs R030 and R019 as measured by a standard order parameter gradient statistic [- [1] that effectively measures the mean distance between interfaces crossing the chosen direction.

In 12], finite size effects (in the absence of shear) were considered quantitatively under control when the correlation length $L$ was less than $1 / 4$ of the system size $\Lambda$. In [6] this criterion was applied to time-averaged correlation lengths $L_{x, y}$ in the 2D sheared system. However, the actual system size dependence of $L_{x, y, z}$ in both 2D [6] and $3 \mathrm{D}$ (this work) suggests that under shear this criterion is unnecessarily strict, at least if the purpose is to eliminate the qualitatively artefactual states that arise directly from finite size effects. As mentioned previously, these "trivial" NESS's form obvious laminar stripes extending the full size of the simulation box in both $x$ and $z$ directions.
For such states, $L_{x, z}$ values that are formally much larger than the simulation dimensions $\Lambda_{x, z}$ are rapidly established. ( $L_{x} \gg \Lambda_{x}$ means that that, for most coordinates $y, z$, one can cycle round the periodic boundary conditions in $x$ without encountering a single domain wall.) To formally eliminate these, a criterion $L_{x, y, z} \leq \Lambda_{x, y, z}$ is applied, which also excludes one apparently nontrivial NESS run (Table 1) from the scaling analysis made below. At the lowest Reynolds numbers investigated, only a trivial NESS was found on a $512 \times 256 \times 256$ lattice; larger systems, $1024 \times 512 \times 512$, were then simulated for these parameters but gave the same structure. This difficulty in achieving bulk NESS at low Re perhaps suggests onset of a new regime; we return to this below.

Only at the largest $\left(\dot{\gamma} T_{0}\right)^{-1}$ values investigated was the strict finite size criterion of [12], $L_{x}<\Lambda_{x} / 4$, approached. (Note however that earlier studies accepted $L<\Lambda / 2$ as sufficient, e.g. [21].) Accordingly we expect that the quantitative scaling of all our correlation length data with shear rate may still be affected by finite size corrections. With this caveat, we proceed to perform a scaling analysis based on the protocol of [6. To construct our scaling plot, mean values of $L_{x, y, z}$ were obtained via a bootstrap procedure [6] performed on each times series, discarding data for which $t<10^{5}$ (to eliminate transients). The results for $L_{x, y, z} / L_{0}$ are plotted against $\left(\dot{\gamma} T_{0}\right)^{-1}$ in Fig. 3. Linear least-squares fits to these data suggest scaling exponents for $L_{x, y, z}$ of, respectively, $-0.54 \pm 0.03,-0.65 \pm 0.03$, and $-0.60 \pm 0.04$ at the $95 \%$ confidence level. An alternative scaling, using the principal axes of the gradient statistic [- [6, gives exponents for $L_{3}, L_{1}, L_{2}$ of $-0.53 \pm 0.04,-0.67 \pm 0.03$, and $-0.64 \pm 0.06$ (data not shown). These results appear to rule out $L_{y} \sim \dot{\gamma}^{-3 / 4}$ which was found in $2 \mathrm{D}$ [6]. However, the range of Re accessible is restricted to about 1 decade $(260 \leq \operatorname{Re} \leq 2300)$; as in $2 \mathrm{D}$, one cannot rule out that these are effective exponents describing the 


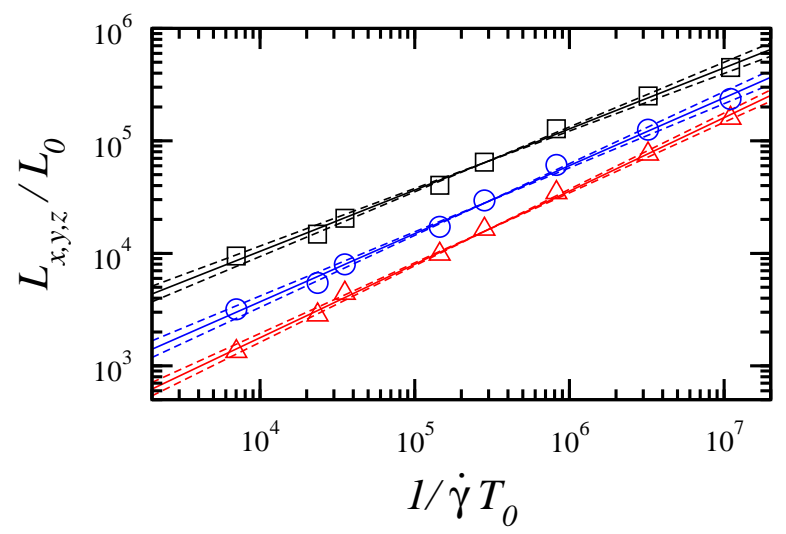

Figure 3: Reduced length scales $L_{x, y, z} / L_{0}$ (black squares, red triangles, blue circles respectively) as a function of inverse reduced shear rate for the 8 runs in which nontrivial NESS was observed. The standard errors in the individual points are no larger than the symbols; the dashed lines give the $95 \%$ confidence limits of the fitted regression.

crossover region. These Re values are also high enough that a multiple length scaling might be needed 22].

The quoted error margins do not, of course, allow for systematic error of which there are several sources (even discounting finite size effects), each at the likely level of several percent 6, 12. Accordingly these results do not rule out a common scaling of all three correlation lengths with a single exponent, $L_{x, y, z} / L_{0} \sim\left(\dot{\gamma} T_{0}\right)^{-2 / 3}$, at least in the inertial limit of very large $\left(\dot{\gamma} T_{0}\right)^{-1}$ where the data hints that the three curves may saturate to fixed ratios. Conversely, the ever increasing difficulty to achieve NESS at small $\left(\dot{\gamma} T_{0}\right)^{-1}$ may point to a quite different behavior at low Reynolds numbers. Suggestively, Fielding 23] has recently performed 2D binary Stokes flow simulations finding no evidence of bulk NESS at Re $=0$; this could mean that inertia plays the role of a singular perturbation in this problem. Moreover, for a range of $\dot{\gamma} T_{0}$ around $10^{-3}$, NESS is easily achieved in 2D but not 3D: the ability to form connections in the vorticity direction might, at moderate and low Re, require formation of domains of extremely high aspect ratio before a NESS can be reached.

In conclusion, while open issues remain concerning the details of scaling and finite size behavior, our simulations present clear evidence for nonequilibrium steady states in $3 \mathrm{D}$ sheared binary fluids. The qualitative character of the NESS achieved in these simulations at high Re (low shear rate), which entails a balance between domain stretching under flow and coarsening driven by interfacial tension, strongly suggests that these results represent true bulk behavior. Since the effect of coarsening at fixed $\dot{\gamma}$ is to increase Re, indefinite coarsening [9] can seemingly be ruled out even at higher shear rates, although a different mechanism for achieving NESS may operate there.

Acknowledgements: Work funded in part by EPSRC EP/C536452 and GR/S10377. JCD acknowledges use of facilities at the Irish Centre for High-End Computing. We thank S. Fielding and R. Adhikari for discussions.
[1] M. E. Cates and M. R. Evans (Eds.), Soft and Fragile Matter, Nonequilibrium Dynamics, Metastability and Flow, IOP Publishing, Bristol 2000.

[2] A. Onuki, Phase Transition Dynamics, Cambridge University Press, Cambridge 2002.

[3] M. E. Cates, V. M. Kendon, P. Bladon and J.-C. Desplat, Faraday Disc. 112, 1 (1999).

[4] A. J. Wagner and J.M. Yeomans, Phys. Rev. E 59, 4366 (1999); Phys. Rev. Lett. 80, 1429 (1998).

[5] A. Lamura and G. Gonnella, Physica A 294, 295 (2001); F. Corberi, G. Gonnella and A. Lamura Phys. Rev. Lett. 81, 3852 (1998); Phys. Rev. Lett. 83, 4057 (1999); Phys. Rev. E 61, 6621 (2000); Phys. Rev. E 62, 8064 (2000).

[6] P. Stansell, K. Stratford, J.-C. Desplat, R. Adhikari, and M. E. Cates Phys. Rev. Lett. 96085701 (2006).

[7] M. E. Cates and S. T. Milner, Phys. Rev. Lett. 62, 1856 (1989).

[8] T. Hashimoto, T. Takebe and S. Suehiro, J. Chem. Phys. 88, 5875 (1988); C. K. Chan, F. Perrot and D. Beysens, Phys. Rev. A 43, 1826 (1991); A. H. Krall, J. V. Sengers and K. Hamano, Phys. Rev. Lett. 69, 1963 (1992); T. Hashimoto, K. Matsuzaka, E. Moses and A. Onuki, Phys. Rev. Lett. 74, 126 (1995); Y. Takahashi, N. Kurashima, I. Noda and M. Doi, J. Rheol. 38, 699 (1994).

[9] A.J. Bray, Phil. Trans. Roy. Soc. A 361, 781 (2003); A. Cavagna, A.J. Bray, and R.D.M. Travasso, Phys. Rev. E 62, 4702 (2000); A. J. Bray and A. Cavagna, J. Phys. A 33, L305 (2000).
[10] A. Onuki, J. Phys. Cond. Mat. 9, 6119 (1997).

[11] M. Doi and T. Ohta, J. Chem. Phys. 95, 1241 (1991).

[12] V.M. Kendon, et al., J. Fluid Mech. 440, 147 (2001); V.M. Kendon, J.-C. Desplat, P. Bladon and M.E. Cates, Phys. Rev. Lett. 83, 576 (1999); I. Pagonabarraga, A.J. Wagner, and M.E. Cates, J. Stat. Phys. 107, 39 (2002).

[13] J. Harting, J. Chin, M. Venturoli and P. V. Coveney, Phil. Trans. Roy. Soc. Lond. A 363, 1895 (2005).

[14] M. E. Cates, et al., Phil. Trans. Roy. Soc. A 363, 1917 (2005).

[15] M. R. Swift, E. Orlandini, W. R. Osborn and J. M. Yeomans, Phys. Rev. E 54, 5041 (1996).

[16] A. J. Wagner and I. Pagonabarraga, J. Stat. Phys. 107, 521 (2002).

[17] A. J. Bray, Adv. Phys. 43, 357 (1994).

[18] D. d'Humières, I. Ginzburg, M. Krafczyk, P. Lallemand and L.-S. Luo, Phil. Trans. R. Soc. A 360, 437 (2002).

[19] K. Stratford, R. Adhikari, I. Pagonabarraga, J.-C. Desplat, J. Stat. Phys. 121, 163 (2005).

[20] An animation of this run is available as supplementary online material.

[21] S. I. Jury, P. Bladon, S. Krishna and M. E. Cates, Phys. Rev. E 59, R2535 (1999).

[22] V. M. Kendon, Phys. Rev. E 61 R6071 (2000).

[23] S. M. Fielding, private communication. 